\title{
Operação de Rastelli utilizando-se conduto valvulado de pericárdio bovino: experiência inicial
}

\author{
Carlos R. MORAES*, Jorge V. RODRIGUES*, Cláudio A. GOMES*, Lorella MARINUCCI*, Cleuza Lapa
} SANTOS*, Tereza Cristina COELHO*, Ivan de Lima CAVALCANTI*

MORAES, C. R.; RODRIGUES, J. V.; GOMES, C. A.; MARINUCCI, L.; SANTOS, C. L.; COELHO, T. C.; CAVALCANTI, I. L. - Operação de Rastelli utilizando-se conduto valvulado de pericárdio bovino: experiência inicial. Rev. Bras. Cir. Cardiovasc., 3(2): 84-92, 1988.

RESUMO: O presente trabalho descreve a experiência inicial com a operação de Rastelli, utilizando-se um conduto valvulado de pericárdio bovino preservado em glutaraldeído. O enxerto consta de um conduto, que serve para reconstruir a via de saída do ventrículo direito e o tronco pulmonar, com uma válvula tricúspide suturada em seu interior sem anel de suporte, permitindo, assim, um mecanismo de fluxo unidirecional. De maio de 1986 a outubro de 1987,5 crianças foram submetidas à operação de Rastelli empregando-se este tipo de conduto valvulado. Três eram do sexo feminino e 2 , do masculino, variando a idade de 1 a 8 anos $\bar{x} 5$ ). Três pacientes tinham transposição das grandes artérias, comunicação interventricular (CIV) e estenose subpulmonar. Uma criança era portadora de atresia pulmonar com CIV e de um shunt prévio tipo Waterston e, finalmente, a outra tinha um tronco arterial comum tipo II. Dois doentes faleceram de causas não relacionadas ao tipo de conduto valvulado utilizado. $O$ conduto valvulado de pericárdio bovino preservado em glutaraldeído mostrou excelente flexibilidade, facilitando o manuseio cirúrgico e permitindo perfeita adaptabilidade dentro do saco pericárdico. A ausência de anel de suporte tem a vantagem de eliminar qualquer gradiente e de abolir o turbilhonamento do sangue - causas reconhecidas de calcificação.

DESCRITORES: condutos valvulados, pericárdio bovino, cirurgia; Rastelli, operação de.

\section{INTRODUÇÃO}

O tratamento cirúrgico de vários tipos de cardiopatias congênitas complexas requer, em uma de suas etapas, a criação de um conduto extracardíaco conectando o ventrículo venoso ao tronco pulmonar. RASTEL$\mathrm{LI}$ et alii ${ }^{19}$, em 1965 , foram os primeiros a usar um tubo não valvulado de pericárdio autógeno em 1 caso de atresia pulmonar. Graças a este procedimento pioneiro, é costume denominar-se operaçāo de Rastelli a todas as cirurgias que empregam condutos extracardiacos para restabelecer a continuidade entre o ventrículo direito e o tronco pulmonar.
Diversos tipos de condutos, valvulados ou não, têm sido utilizados nos últimos 20 anos, destacando-se os homoexertos aórticos ${ }^{20}$ e os tubos de Dacron, contendo uma válvula heteróloga de porco em seu interior ${ }^{2,}{ }^{17}$. Todos estes condutos têm apresentado problemas na evolução tardia ${ }^{1}, 3,9,12,14,16,22$, justificando-se, assim, as pesquisas para a obtenção de um tubo valvulado ideal ${ }^{7}$. 15. 21 .

Em 1970, IONESCU \& DEAC ${ }^{8}$ descreveram uma engenhosa técnica de preparação de um conduto valvulado com fascia lata, mas este tecido cedo se mostrou inadequado como enxerto em cirurgia cardiovascular.

Trabalho realizado no Instituto do Coraçào de Pernambuco. Real Hospital Português de Beneficéncia em Pernambuco. Recife. PE. Brasil. Apresentado ao 15: Congresso Nacional de Cirurgia Cardiaca. Rio de Janeiro, RJ, 8 e 9 de abril, 1988.

- Do Instituto do Coração de Pernambuco. Real Hospital Português de Beneficiência em Pernambuco.

Endereço para separatas: Carlos R. Moraes. Av. Bernardo Vieira de Melo. 4405/491. Jaboatâo. 56.100 Recife, PE, Brasil 
MORAES, C. R.; RODRIGUESL, J. V.; GOMES, C. A.; MARINUCCI, L.; SANTOS, C. L.; COELHO, T. C.; CAVALCANTI, I. L. - Operação de Rastelli utilizando-se conduto valvulado de pericárdio bovino: experiência inicial. Rev. Bras. Cir. Cardiovasc. 3(2): 84-92, 1988.

Recentemente, decidimos confeccionar, industrialmente, com base na técnica de lonescu e Deac, um conduto valvulado inteiramente feito de pericárdio bovino, material este que tem demonstrado boas qualidades como enxerto em cirurgia cardiovascular ${ }^{4}$. A experiência inicial com este novo tipo de tubo valvulado constitui o motivo do presente trabalho.

\section{CASUISTICA E MÉTODOS}

De maio de 1986 a outubro de 1987, no Instituto do Coração de Pernambuco, 5 pacientes foram submetidos á operaçâo de Rastelli, utilizando-se um conduto valvulado de pericárdio bovino, conservado em glutaraldeido (Tabela 1). Três crianças eram do sexo feminino

TABELA 1

\begin{tabular}{clcccc}
\hline CASO & \multicolumn{1}{c}{ DIAGNÓSTICO } & $\begin{array}{c}\text { IDADE } \\
\text { (anOS) }\end{array}$ & $\begin{array}{c}\text { PESO } \\
(\mathrm{kg})\end{array}$ & $\begin{array}{c}\text { DIAMETRO TUBO } \\
\text { (mm) }\end{array}$ & RESULTADO \\
\hline 1 & TGA + CIV + EP & 8 & 21 & 20 & Bom \\
2 & TGA + CIV + EP & 8 & 14 & 20 & Bom \\
3 & Atresia pulmonar + CIV + shunt & 8 & 16 & 20 & Obito \\
4 & TAC tipo II & 1 & 7 & 18 & Obito \\
5 & TGA + CIV + EP & 2 & 12 & 22 & Bom \\
\hline
\end{tabular}

$\mathrm{TGA}=$ transposição das grandes artérias; $\mathrm{CIV}=$ comunicação interventricular; $\mathrm{EP}=$ estenose pulmonar; $\mathrm{TAC}=$ tronco arterial comum.

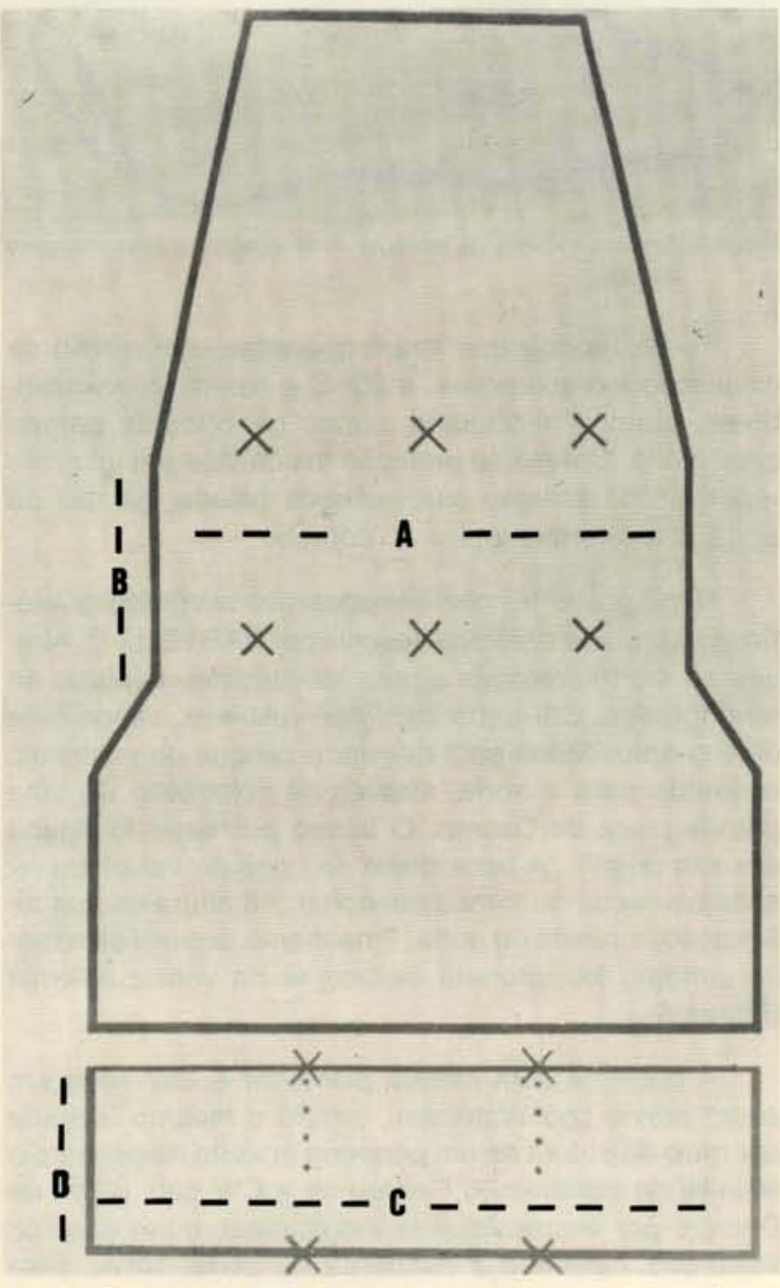

Fig. 1 - Formato dos segmentos a serem recortados da membrana de pericárdio e a marcação dos pontos comissurais. e 2, do masculino, variando a idade de 1 a 8 anos $(\bar{x}$ 5). O peso variou de 7 a $31 \mathrm{~kg}(\bar{x} 14)$. Três pacientes tinham transposição das grandes artérias, comunicação

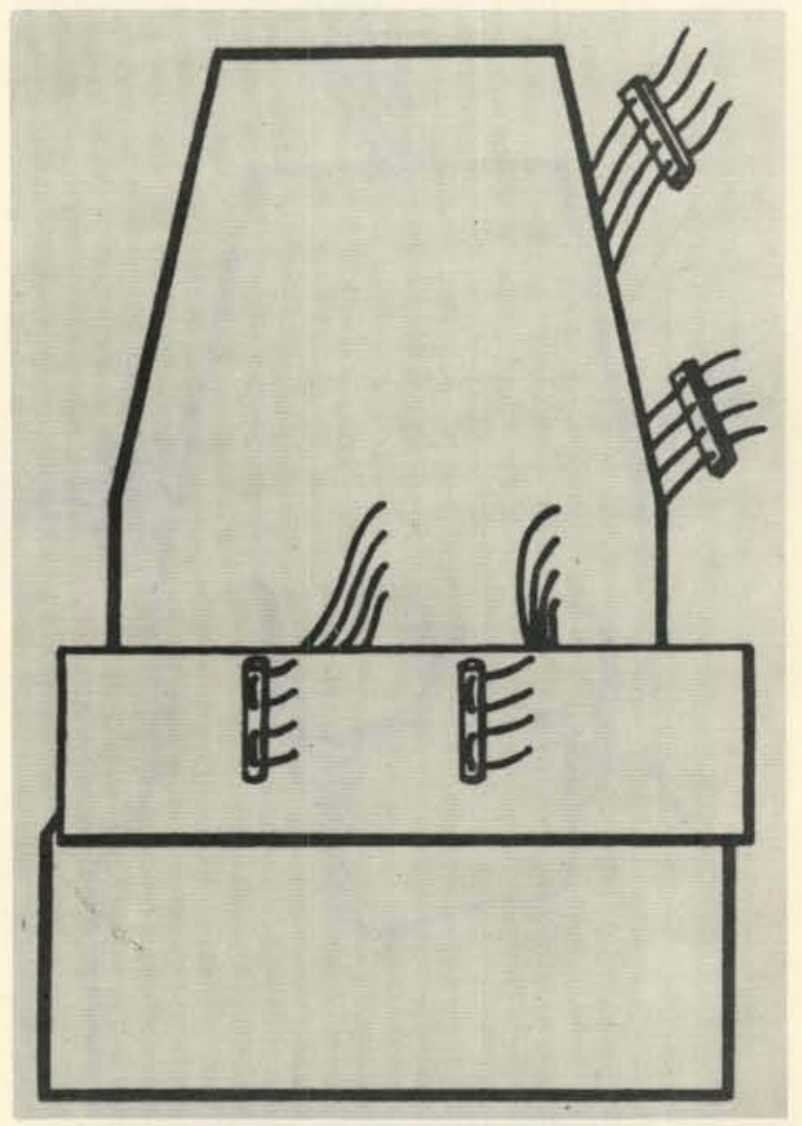

Fig. 2 - Fixaçāo do segmento retangular no local dos pontos comissurais. 
MORAES, C. R.; RODRIGUESL, J. V.; GOMES, C. A.; MARINUCCI, L.; SANTOS, C. L.; COELHO, T. C.; CAVALCANTI, I. L. - Operação de Rastelli utilizando-se conduto valvulado de pericárdio bovino: experiência inicial. Rev. Bras. Cir. Cardiovasc. 3(2): 84-92, 1988.

interventricule- (CIV) e estenove subpulmonar; 1 possuia atresia pulmonat com CIV e um shunt prévio tipo Waterston e o outro, tronco arterial comum tipo II. O diagnóstico foi estabelecido por estudo clínico, ecocardiográfico e angiocardiográfico, sendo a indicação cirúrgica realizada pela presença de severa hipoxemia e/ou insuficiência cardiaca.

A técnica de preparação do conduto valvulado de pericárdio bovino já foi previamente descrita em detaInes ${ }^{13}$. Obtém-se o conduto valvulado de uma membrana de pericárdio bovino, fixada em glutaraldeído e conservada em formaldeido. Sāo cortados 2 segmentos desta membrana (Figura 1), obedecendo-se aos tamanhos relacionados na Tabela 2 . O diâmetro interno dos tubos varia de 12 a $24 \mathrm{~mm}$, destinando-se o segmento maior à confecção do conduto, e o segmento retangular, à preparação da válvula. Os pontos comissurais são marcados (Figura 1) e o segmento retangular é fixado no conduto, inicialmente ao nivel de 2 comissuras (Figura 2). As 2 bordas do segmento retangular são suturadas juntas, formando-se um cilindro, para, após, serem fixadas no local da terceira comissura (Figura 3 ). No topo de cada comissura, dá-se um ponto, a fim de fechar a válvula na posição de repouso (Figura 3 ). As margens

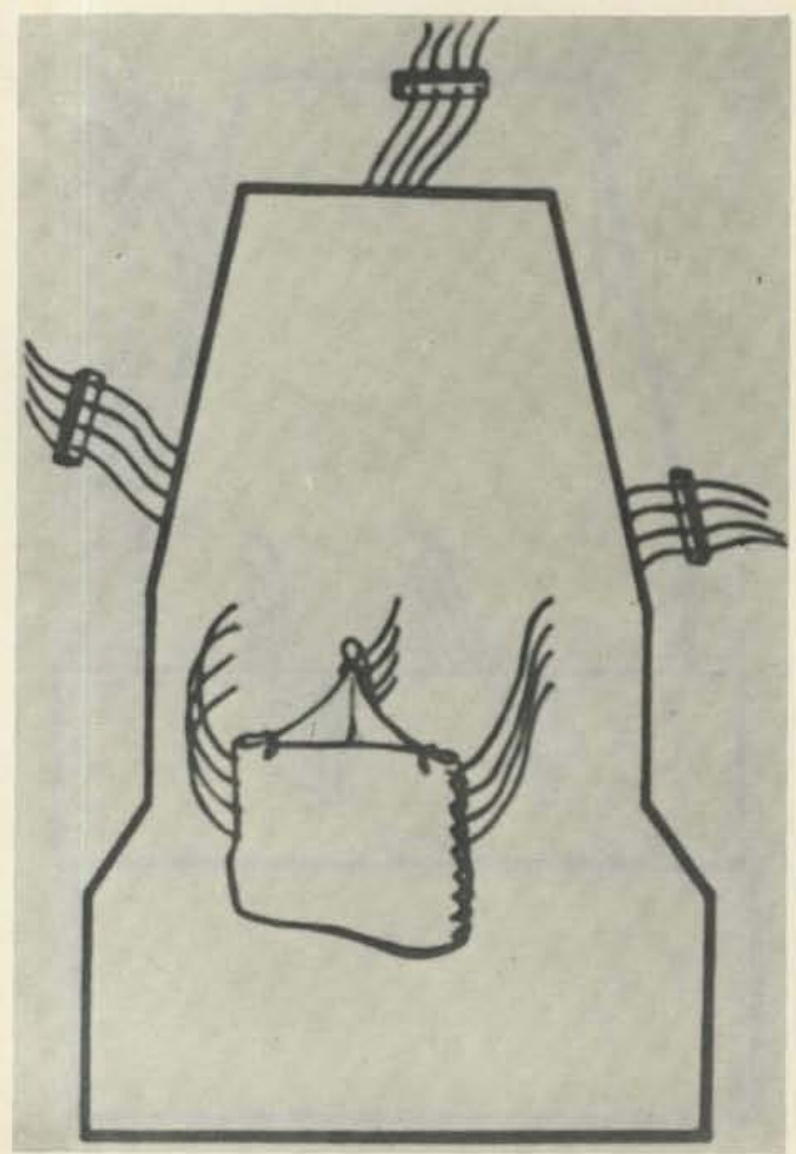

Fig. 3 - Sutura das bordas do segmento retangular, formando um cilindro. Fixação no local da terceira comissura.
TABELA 2

DIMENSÓES DASLAMINAS DE PERICARDIO BOVINO $(\mathrm{mm})$

\begin{tabular}{llllllll}
\hline D.I. & 12 & 14 & 16 & 18 & 20 & 22 & 24 \\
\hline A & 45 & 50 & 55 & 60 & 65 & 70 & 75 \\
B & 12 & 13,5 & 15 & 16,5 & 18 & 19,5 & 21 \\
C & 55 & 60 & 65 & 70 & 75 & 80 & 85 \\
D & 12 & 13,5 & 15 & 16,5 & 18 & 19,5 & 21 \\
\hline
\end{tabular}

D.I. = Diâmetro interno.

laterais do segmento maior são, entāo, suturadas, fechando-se, assim, o conduto (Figura 4). A parte proximai do conduto poderá ser recortada durante a operação, de tal sorte a adaptá-la ao tamanho da ventriculotomia. A preparação do tubo valvulado é concluída, amarrando-se as suturas comissurais por fora do conduto e suturando-se a base da válvula ao tubo. Estes condutos valvulados sāo, atualmente, manufaturados em diversos diâmetros e tamanhos *

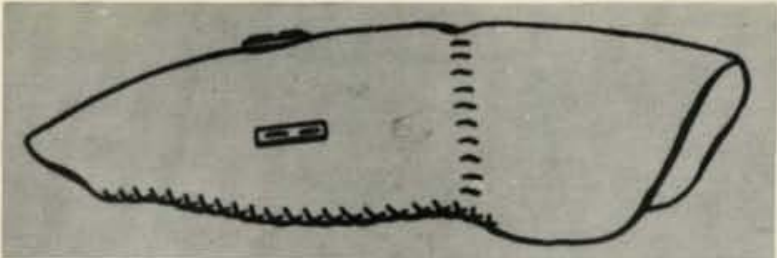

Fig. 4-Aspecto externo do conduto após sutura de suas margens laterais.

Todas as crianças foram operadas com auxilio da circulação extracorpórea, a $20^{\circ} \mathrm{C}$ e hipofluxo, realizando-se, quando necessário, curtos períodos de parada circulatória. Obteve-se proteção miocárdica por infusões repetidas de solução cardioplégica gelada, na raiz da aorta, e hipotermia tópica do coração.

Nos 3 pacientes com transposiçāo das grandes artérias, realizou-se a técnica descrita por RASTELLI ${ }^{18}$. Através de ventriculotomia direita longitudinal, verificou-se terem, todos, CIV subaórtica não restritiva, sendo este defeito aproveitado para desviar o sangue do ventrículo esquerdo para a aorta, através da colocação de uma grande placa de Dacron. O tronco pulmonar foi ligado em sua origem. A boca distal do conduto valvulado foi anastomosada ao tronco pulmonar, na altura de sua bifurcação, à direita da aorta. Finalmente, a parte proximal do conduto foi suturada às bordas da ventriculotomia (Figura 5).

A paciente com atresia pulmonar e CIV tinha um shunt prévio tipo Waterston, sendo o mesmo fechado por meio da sutura de um pequeno enxerto de pericárdio através de aortotomia. Fechou-se a CIV com placa de Dacron, por ventriculotomia longitudinal, e um conduto valvulado, colocado à esquerda da aorta, serviu para

\footnotetext{
- Ciconol - Cardio Industrial e Comercial do Nordeste Ltda
} 
MORAES, C. R.; RODRIGUESL, J. V.; GOMES, C. A.; MARINUCCI, L.; SANTOS, C. L.; COELHO, T. C.: CAVALCANTI, I. L. - Operação de Rastelli utilizando-se conduto valvulado de pericárdio bovino: experiência inicial. Rev. Bras. Cir. Cardiovasc., 3(2): 84-92, 1988

restabelecer a continuidade entre o tronco pulmonar $\mathrm{e}$ o ventriculo direito.

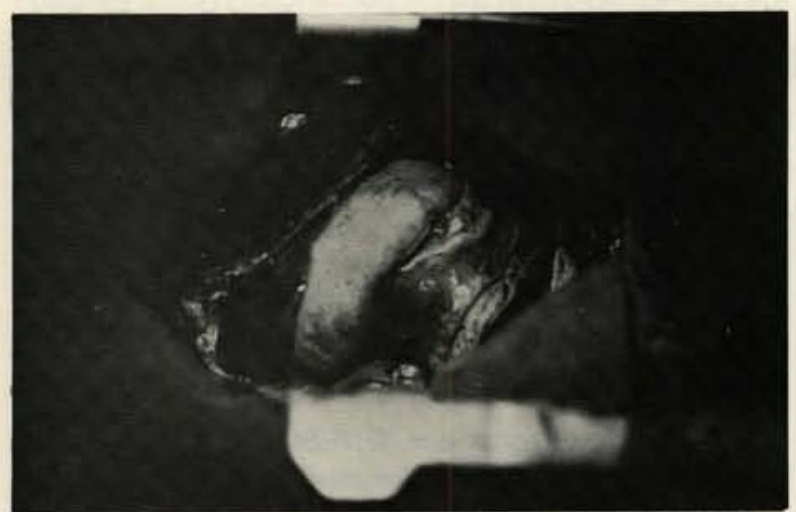

Fig. 5 - Aspecto cirúrgico do conduto valvulado, colocado à direita da aorta, num caso de transposição das grandes artérias.

Finalmente, na criança portadora de tronco arterial comum tipo II, realizou-se uma dupla secção da aorta, acima e abaixo da emergência dos 2 ramos pulmonares, ficando estes 2 vasos conectados por uma parte de parede posterior da aorta. Sua confluência foi, então, obtida pela sutura de uma placa de enxerto de Gortex. A continuidade da aorta foi restabelecida por anastomose término-terminal dos seus cotos. Fechou-se, então, a CIV por ventriculotomia direita longitudinal e, concluindo-se a operaçāo, um conduto valvulado foi interposto entre a artéria pulmonar esquerda e a ventriculotomia direita (Figura 6).

$\mathrm{Na}$ Tabela 1, pode-se observar o diâmetro dos condutos valvulados usados nessa série.

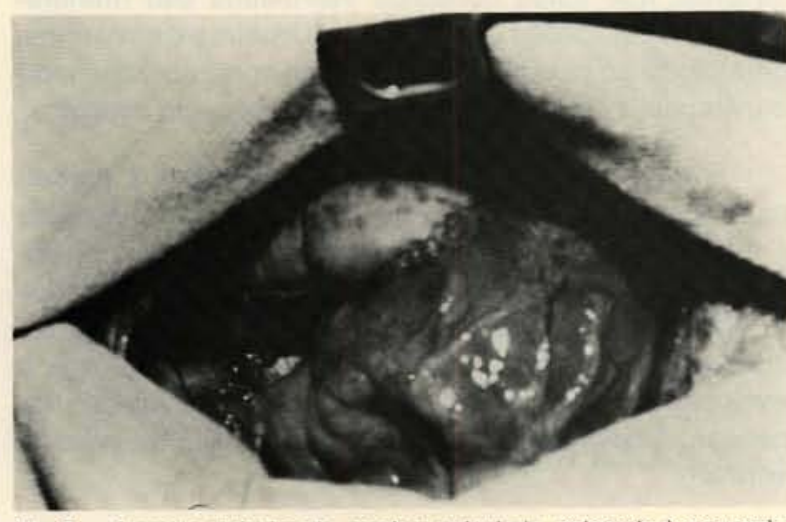

Fig. 6 - Aspecto cirúrgico do conduto valvulado, colocado à esquerda da aorta, num caso de tronco arterial comum.

\section{RESULTADOS}

Ocorreram 2 óbitos, durante a fase de internaçāo hospitalar (Tabela 1). Uma criança apresentou, a partir do 5 : dia de pós-operatório, grave quadro de insuficiência cardiaca em decorrência de reabertura da CIV. A reope- ração, realizada no 8 : dia de evolução, foi feita com a paciente em precárias condiçōes hemodinâmicas, e o óbito sobreveio por insuficiência miocárdica. A outra criança faleceu no 15 . dia de pós-operatório, devido a grave lesão cerebral, mas, até então, manteve boa situação hemodinâmica.

Os 2 primeiros pacientes operados apresentam excelente evoluçāo clínica, decorridos, respectivamente, 19 e 17 meses de cirurgia. O reestudo hemodinâmico, nestes casos, mostrou um excelente aspecto do conduto valvulado, não havendo nenhuma evidência de obstrução (Figuras 7 e 8).

Finalmente, 1 criança, operada há quatro meses, foi reoperada, devido a reabertura da CIV, tendo boa evolução pós-operatória.
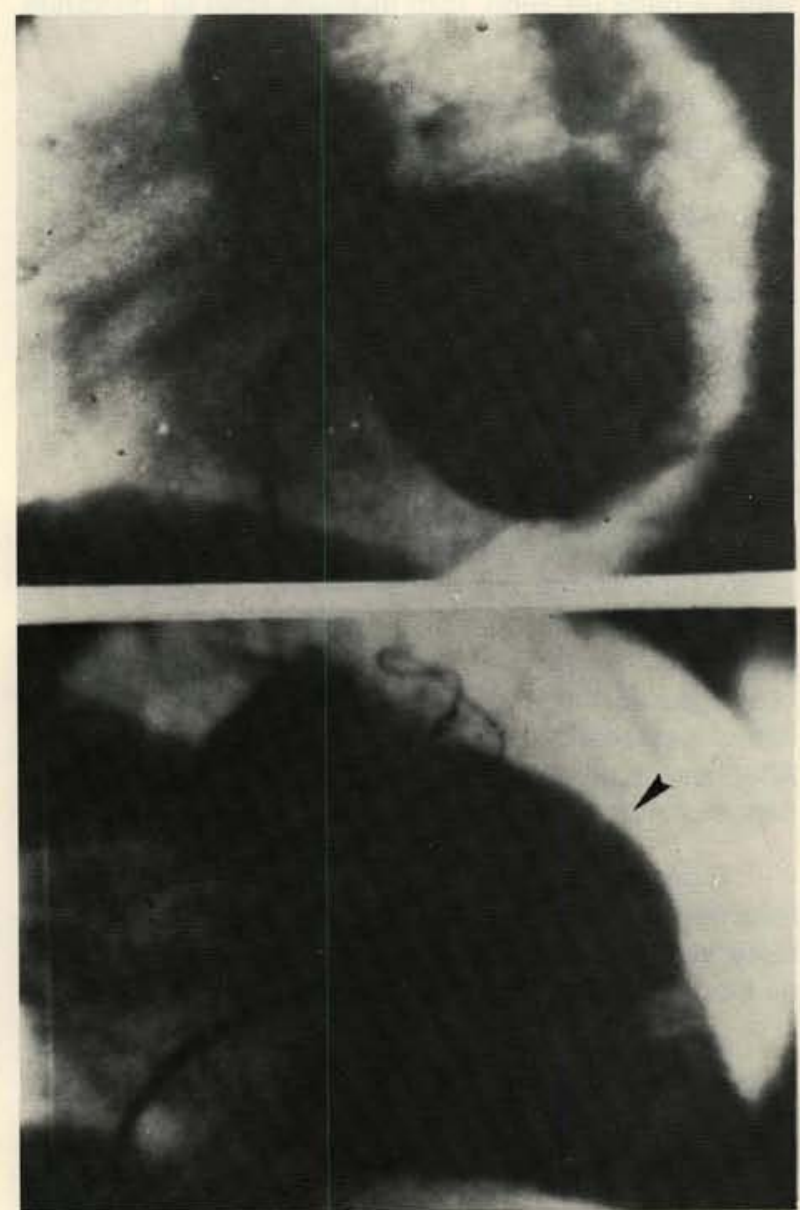

Fig. 7 - Aspecto angiográfico pós-operatório do caso 1. A seta indica - local da válvula.

\section{DISCUSSĀO}

A reconstrução da via de saida do ventrículo direito em cardiopatias congênitas complexas vem sendo reali- 
MORAES, C. R.; RODRIGUESL, J. V.; GOMES, C. A.; MARINUCCI, L.; SANTOS, C. L.; COELHO, T. C.; CAVALCANTI, I. L. - Operação de Rastelli utilizando-se conduto valvulado de pericárdio bovino: experiência inicial. Rev. Bras. Cir. Cardiovasc., 3(2): 84-92, 1988.

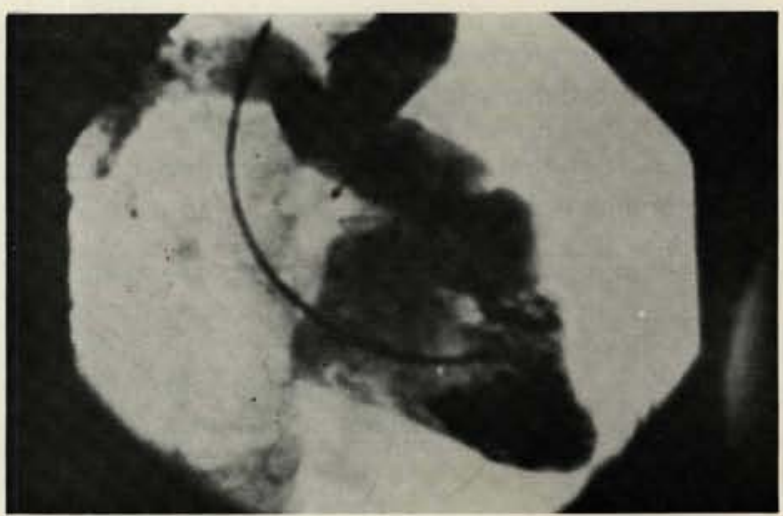

Fig. 8-Aspecto angiográfico pós-operatório do caso 2. Observa-se a ausência de zonas de estenose.

zada, a partir de 1965 , com diversos tipos de condutos extracardiacos.

A experiência com os homoenxertos aórticos preparados por esterilizaçāo química e irradiação foi desapontadora $^{9}, 12,14,{ }^{16}$. KAPLAN et alii ${ }^{9}$ observaram calcificação da valva no primeiro ano após o implante, em 10 dos 22 pacientes que receberam homoenxertos conservados. Melhores resultados tardios vêm, porém, sendo obtidos com os homoenxertos frescos, ou criopreser-

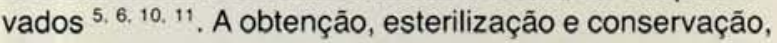
porém, destes enxertos implica problemas adicionais importantes.

A industrialização de condutos de Dacron, contendo uma válvula heteróloga de porco conservada em glutaraldeído, representou um grande avanço, parecendo ser a solução definitiva do problema. Entretanto, os resultados tardios têm revelado uma significativa incidência de obstrução do tubo 1. 14.22. As valvas tornam-se calcificadas e estenóticas, especialmente na idade pediátrica. Ademais, os tubos de Dacron desenvolvem progressiva deposição de fibrina, torrando-se oclusivos.

Tubos avalvulados têm sido utilizados com menos freqüência, uma vez que a insuficiência pulmonar resultante leva a uma lenta, mas progressiva, deterioração da função ventriculari direita ${ }^{21}$.

Verifica-se, por conseguinte, que um conduto extracardiaco ideal está longe de ser obtido, justificando-se as pesquisas com outros tipos de tubo. Condutos valvulados construídos com pericárdio autógeno foram descritos por HORIUCHI et alii ${ }^{7}$ e por SHLICHTER e KREUT$Z_{Z R}{ }^{21}$. Embora a experiência seja pequena e o tempo de evolução pós-operatória, curto, tem sido sugerido que o pericárdio autógeno apresenta potencialidade de crescimento $^{21}$, o que, se for confirmado, representará uma grande vantagem. Mais recentemente, OLIVEIRA et alii ${ }^{15}$ utilizaram pericárdio bovino para confeccionar uma válvula tricúspide dentro de um tubo knitted, cujas maIhas permitiriam uma melhor fixação da neo-íntima. A evolução de 8 dos 9 pacientes operados com esta técnica é muito satisfatória.

A técnica descrita por IONESCU \& DEAC ${ }^{8}$, para confecção de um conduto valvulado sem anel de suporte, é bastante engenhosa, devendo-se a falta de popularidade do método, provalvemente, ao uso de fascia lata, tecido que, rapidamente, se deteriora, quando usado como enxerto no sistema cardiovascular. Decidimos, então, reviver esta técnica, utilizando pericárdio bovino conservado em glutaraldeído, tecido que se tem revelado um bom enxerto na correção de cardiopatias congêni$\operatorname{tas}^{4}$.

Este tipo de conduto valvulado pode oferecer algumas vantagens. A flexibilidade do pericárdio bovino torna o manuseio cirúrgico do conduto mais fácil que o dos tubos de Dacron. Ademais, pareceu-nos haver melhor adaptabilidade do conduto dentro do saco pericárdico. Finalmente, a ausência de anel de suporte tem a vantagem de eliminar qualquer gradiente e de abolir o turbilhonamento do sangue, fatores que, sabidamente, condicionam o aparecimento de calcificaçāo. Deve-se, ainda, salientar que estes condutos valvulados são manufaturados e estocados em diversos diâmetros e tamanhos, permitindo ao cirurgião a escolha do conduto adequado e evitando a confecção do tubo durante $o$ ato cirúrgico.

Evidentemente, nossa experiência inicial é pequena, e o tempo de evolução dos pacientes sobreviventes é muito curto, para que se possa tirar qualquer conclusão. Os dois óbitos ocorridos nāo podem ser relacionados ao tipo de conduto valvulado utilizado. Resta-nos esperar que esta experiência preliminar seja ampliada e que a evolução, a longo prazo, dos condutos valvulados de pericárdio bovino, conservado em glutaraldeido, seja favorável. 
MORAES, C. R.; RODRIGUESL, J. V.; GOMES, C. A.; MARINUCCI, L.; SANTOS, C. L.; COELHO, T. C.; CAVALCANTI, I. L. - Operação de Rastelli utilizando-se conduto valvulado de pericárdio bovino: experiência inicial. Rev. Bras. Cir. Cardiovasc. 3(2): 84-92, 1988.

RBCCV $44205-53$

MORAES, C. R.; RODRIGUES, J. V.; GOMES, C.A.; MARINUCCI, L.; SANTOS, C. L.; COELHO, T. C.; CAVALCANTI, I. L. - Rastelli operation with bovine pericardium valved conduit: early experience. Rev. Bras. Cir. Cardiovasc., 3(2): 84-92, 1988.

ABSTRACT: The present report describes the initial experience with the Rastelli operation utilizing a composite graft of glutaraldehyde preserved bovine pericardium. The graft is made up of a tube which serves to reconstruct the outflow tract of the right ventricle and the pulmonary artery, and an inner tricuspid valve, without a supporting ring, which provides the tube with a one-way flow mechanism. From May, 1986 to October, 1987, five children were submitted to Rastelli operation utilizing this valved tube. Three were female and two male, ranging in age from 1 e to 8 years (mean 5 years). Three had transposition of the great arteries, VSD and subpulmonic stenosis, one had pulmonary atresia, VSD and a previous Waterston shunt, and one had a type II truncus arteriosus. Two patients died of causes unrelated to the type of the conduit used. The valved conduit of preserved bovine pericardium is easy to handle due to its excellent flexibility, allowing perfect adaptability inside the pericardial sac. The absence of a supporting ring abolish gradient across the conduit and turbulence which are recognized causes of calcification.

DESCRIPTORS: valved conduits, bovine pericardium, surgery; Rastelli operation.

\section{REFERÊNCIAS BIBLIOGRÁFICAS}

1 BAYLEY, W. W.; KIRKLIN, J. W.; BARGERON, L. M.; PACIFICO, A. D.; KOUCHOUKOS, N. T. - Late results with synthetic valved external conduits from venous ventricle to pulmonary arteries. Circulation, 56 (Supl. 2): $73-79,1977$.

2 BOWMAN Jr., F. O.; HANCOCK, W. D.; MALM, J. R. A valve containing Dacron prosthesis: its use in restoring pulmonary artery-right ventricular continuity. Arch. Surg., 107(5): 724-728, 1973.

3 BROCK, L. - Long term denegenative changes in aortic segment homograft with particular reference to calcification. Thorax, 23(3): 249-253, 1968.

4 CRAWFORD, F. A.; SADE, R. M.; SPINALE, F. - Bovine pericardium for correction of congenital heart defect. Ann. Thorac. Surg., 41(6): 602-605, 1986.

5 DiCARLO, D.; STARK, J.; REVIGNAS, A.; DelEVAL, M. R. - Conduits containing antibiotic preserved homografts in the treatment of complex congenital heart defects. In: COHN, L. H. \& GALLUCCI, V. eds. Cardiac bioprostheses. New York, Yorke Medical Books, 1982. p. 259-265.

6 FONTAN, F.; CHOUSSAT, A.; DEVILLE, C.; DOUTREMEPUICH, C.; COUPILLAUD, J.; VOSA, C. - Aortic valve homografts in the surgical treatment of complex cardiac malformations. J. Thorac. Cardiovasc. Surg., 87(5): $649-657,1984$

7 HORIUCHI, T.; TADAAKI, A.; OKADA, Y.; KURIBAYASHI, R.; SUZUKI, Y.; ISHIZAWA, E. - Reconstruction of the main pulmonary artery with a valve-bearing tube graft made of autologous pericardium. J. Thorac. Cardiovasc. Surg., 62(5): 793-802, 1971.

8 IONESKU, M. I. \& DEAC, R. C. - Fascia lata composite graft for right ventricular outflow tract and pulmonary artery reconstruction: surgical technique. Thorax, 25(7): 427-435, 1970 .

9 KAPLAN, S.; MCKINIVAN, E.; HELMSWORTH, J. A.; BENZING, F.; SCHWARTZ, D. C.; SCHREIBER, J. T. Complications following homograft replacement of the right ventricular outflow tract. Ann. Thorac. Surg., 18(3): 250-159, 1974.

10 KAY, P. H. \& ROSS, D. N. - Fifteen years experience with the aortic homograft: the conduit of choice for right ventricular outflow tract reconstruction. Ann. Thorac. Surg., 40(4): 360-364, 1985.

11 KIRKLIN, J. W.; BLACKSTONE, E. H.; MAEHARA, T.; PACIFICO, A. D.; KIRKLIN, J. R.; POLLOCK, S.; STEWART, R. W. - Intermediate-term fate of cryopreserved allograft and xenograft valved conduits. Ann. Thorac. Surg., 44(6): 598-606, 1987.

12 MERIN, G. \& McGOON, D. C. - Reoperation after insertion of aortic homograft as a right ventricular outflow tract. Ann. Thorac. Surg., 16(2): 122-126, 1973.

13 MORAES, C. R. \& RODRIGUES, J. V. - Conduto valvulado de pericárdio bovino: relato de caso. Arq. Bras. Cardiol., 48(3): 173-177, 1987.

14 NORWOOD, W. I.; FREED, M. D.: ROCCHINI, A. P.; BERNARD, W. F.; CASTAÑEDA, A. R. - Experience with valved conduits for repair of congenital cardiac lesions. Ann. Thorac. Surg., 24(3): 223-232, 1977.

15 OLIVEIRA, J. B.; SOUZA, L. C. B.; ARNONI, A. S.; SILVEIRA, F. S.; FIGUEIREDO, L. F.; CONFORTI, C. A.; BEMBOM, M. C.; JATENE, A. D.; PAULISTA, P. P. - Conduto valvulado: nova técnica. Arq. Bras. Cardiol., 46(6): 401-406, 1985.

16 PARK, S. C.; NECHES, W. H.; LENOX, C. C.; ZUBERBUHLER, J.R.; BAHSON, H. T. - Massive calcification and obstruction in a homograft after the Rastelli pro- 
MORAES, C. R.; RODRIGUESL, J. V.; GCMES, C. A.; MARINUCCI, L.; SANTOS, C. L.; COELHO, T. C.; CAVALCANTI, I. L - Operação de Rastelli utilizando-se conduto valvulado de pericárdio bovino: experiência inicial. Rev. Bras. Cir. CardiovasC. 3(2): 84-92, 1988.

cedure for transposition of the great arteries. $A m$. J. Cardiol., 32(5): 860-863, 1973.

17 PLANCHE, C.; BINET, J. P; LANGLOIS, J.; CONSO, J. F. - Reconstruction de la voie d'éjection du ventricule droit à l'aide de tubes valvés: problèmes techniques. Nouv. Presse Méd., 1: 541-542, 1972.

18 RASTELLI, G. C. - A new approach to anatomic repair of transposition of the great arteries. Mayo Clin. Proc., 44(1): 1-12, 1969.

19 RASTELLI, G. C.; ONGLEY, P. A., DAVIS, G. D.; KIRKLIN, J. W. - Surgical repair for pulmonary valve atresia with coronary pulmonary artery fistula: report of a case. Mayo Clin. Proc., 40(7): 521-527, 1965.

20 ROSS, D. N. \& SOMERVILLE, J. - Correction of pulmonary atresia with a homograft aortic valve. Lancet. 2(7479): 1446-1447, 1966.

21 SCHLICHTER, A. J. \& KREUTZER, G. O. - Tubo valvulado de pericárdio autólogo. Rev. Lat. Cardiol. Cir. Cardiovasc. Infantil, 1(1): 37-42, 1985.

STEWART, S.; MANING, J.; ALEXSON, C.; HARRIS, P. - The Hancock external valve conduit. J. Thorac. Cardiovasc. Surg., 86(4): 562-569, 1983.

\section{Discussão}

\section{DR. FÁBIO SALLUM Curitiba, $P R$}

O Dr. Donald Ross, em 1966, realizou, pela primeira vez, a colocação de um homoenxerto aórtico para a correção de uma atresia pulmonar, sendo descrita em artigo publicado no Lancet, no mesmo ano. O Dr. Rastelli publicou sua experiência inicial com o uso de tubos valvulados para a correção de truncus arteriosus, em 1969. no Journal of Thoracic and Cardiovascular Surgery. Durante o Il: Simpósio Internacional de Cirurgia Cardiaca, do Hospital Henry Ford, o Dr. Dwight Harken citou, na palestra de abertura, as contribuiçōes mais importantes de alguns cirurgiōes cardiovasculares. Referindo-se ao Dr. Donald Ross, o Dr. Harken descreveu: "Realizou a reconstrução da via de saida do ventrícuto direito com tubo valvulado antes de Rastelli, em 1966. Entendemos de justiça, portanto, a denominação desta operação como "operação de Ross". A utilização de conduto valvulado, mesmo sem suporte, de pericárdio bovino, nos pareceu satisfatória, na experiência do Dr. Carlos Moraes. Estes resultados iniciais, entretanto, são superponiveis a outros materiais empregados, provavelmente pelo curto período de observação. A experiência do emprego de tubo valvulado porcino, de $12 \mathrm{~mm}$, em recémnatos, na Universidade da Califórnia, demonstrou a necessidade de reoperaçāo em $67 \%$ dos sobreviventes, em um período de 10 anos, por estenose relativa do el:xorto; $37 \%$ destes pacientes apresentaram elevada pressāo no ventrículo direito, no mesmo período de observação. Para analisarmos a real efetividade do conduto valvulado de pericárdio bovino proposto neste trabalho, deveremos aguardar periodo semelhante ao do grupo da Universidade da Califórnia. Um fator de melhor prognóstico, no grupo do Dr. Carlos Moraes, foi o fato de ter empregado diâmetros provavelmente maiores, já que seus pacientes eram de faixa etária superior. Obrigado.

\section{DR. JOSÉ ALDROVANDO DE OLIVEIRA Rio de Janeiro, RJ}

O trabalho do Dr. Carlos Roberto Moraes e colaboradores, original e de primeira plana, representa um marco na procura do conduto ideal para a realização da operaçāo de Rastelli. Todavia, dentro do espírito científico, torna-se necessário, para melhor quantificar mais esta contribuiçāo da Escola do Recife, uma avaliação dos resultados a longo prazo em número maior de cirurgias. A supressão do anel da bioprótese - já realizada, entre nós, pelo grupo cirúrgico do Instituto Dante Pazzanese de Cardiologia, a partir de 1981, como modificação do clássico tubo de Hancock, inclusive, com utilização de pericárdio bovino para a constituição das cúspides afigura-se como de maior importância para a profilaxia da obstrução deste tipo de derivação extracardiaca, haja visto pripiciar: amenização do gradiente transvalvular e redução marcante do turbilhonamento do fluxo sangüineo e, conseqüentemente, menor possibilidade de ocorrência tardia de calcificação. Cumpre rememorar que o restabelecimento da continuidade vascular, através da utilização de anéis rígidos, desde a prótese perdida de magnésio, idealizada por Payr, em 1901, até o tubo de vitálio disposto por fora das extremidades do enxerto venoso, recomendado por Bakemore e Lord, em 1942 , fez-se acompanhar de obstruçāo, como demonstrou, entre outros, Wanderley Filho, em 1947, em tese apresentada á Faculdade de Medicina do Recife, quando evidenciou, em cães, com a técnica de Blakemore, oclusão do enxerto entre o 6 : e o 16 : dia de pós-operatório. em decorrência, sobretudo, dos estudos desenvolvidos pỏr Braile e colaboradores, no Instituto de Moléstias Cardiovasculares, de São José do Rio Preto, ampliam-se, entre nós, as indicaçōes do uso da membrana peridárdica bovina, em cirurgia cardiaca. Pelas suas características de resistência, impermeabilidade, flexibilidade e grau minimo de antigenicidade, quando fixada em solução de glutaraldeído, como estabeleceu Carpentier, em 1969 , acreditamos ser o pericárdio bovino suficientemente adequado, como propōem Moraes e colaboradores, para a elaboração do tubo valvulado, em toda sua extensão. Os autores foram extremamente felizes, ao utilizarem a técnica de lonescu e Deac, para o preparo do conduto valvulado, o que torna o método simples e, conseqüentemente, ao alcance dos demais Centros, 
MORAES, C. R.; RODRIGUESL, J. V.; GOMES, C. A.; MARINUCCI, L.; SANTOS, C. L.; COELHO, T. C.: CAVALCANTI, I. L. - Operaçâo de Rastelli utilizando-se conduto valvulado de pericárdio bovino: experiência inicial. Rev. Bras. Cir. Cardiovasc. 3(2): 84-92, 1988.

ao contrário do conduto valvulado de pericárdio de porco idealizado por Rygg, no Departamento de Cirurgia Cardiotorácica do Hospital Universitário de Copenhagen, em que o pericárdio, relativamente fino $(0,10$ a $0,15 \mathrm{~mm})$, é envolvido em tecido de jérsei e nele fixado por uma série de suturas transversais. A experiência adquirida no Instituto de Cardiologia Aloysio de Castro, com o uso do pericárdio autógeno e bovino em outras técnicas cirúrgicas, dá-nos a convicção de que o conduto idealizado pela Escola Cirúrgica do Recife apresenta imensas vantagens, quando ao manuseio cirúrgico, propiciando sutura fácil e hemostática, além de melhor adaptação à cavidade mediastínica em relaçâo ao tubo de Hancock, tão propenso a espessamentos e descolamentos tardios da intima no interior do Dacron, ou nas bocas anastomóticas, salvo quando se utilize tubo Knitted pré-coagulado, cujas malhas mais amplas permitem o crescimento firme das pontes fibrosas para o interstício do tubo, proporcionando maior estabilidade da neo-íntima. Desejo parabenizar o Dr. Carlos Moraes e sua equipe, pelo excelente trabalho apresentado.

\section{DR. DOMINGO BRAILE \\ São José do Rio Preto, SP}

Apenas os parabéns ao Carlos Moraes. Eu já havia comentado com ele antes, que o pericárdio permite uma porção de criatividade e isto é um benefício grande para os pacientes; apenas para lembrar que nós temos, já há 7 anos, os tubos valvulados com suporte, evidentemente na posição aórtica, de aorta ascendente, e não temos nenhum caso em que esse tubo teve que ser trocado; temos seguido esses pacientes, com ecocardiografia, e alguns deles foram submetidos a angiocardiografia e, agora, estão sendo seguidos por ecocardiografia e apresentam uma evolução muito boa. Tenho certeza, portanto, que o tubo do lado direito ainda vai se comportar muito melhor, com certeza não vai haver o problema da formação de um anel íntimo, com proliferação e, muito provavelmente, esse tubo terá uma longa duração. Parabenizo, novamente, o Carlos, pelo trabalho e pela criatividade demonstrada.

PROF. ADIB D. JATENE

São Paulo, SP

Eu queria cumprimentar o Dr. Carlos Moraes pelo trabalho. Como foi mostrado pelo Dr. João Bosco, nós utilizamos, em casos de truncus, o tubo de Dacron com válvula de pericárdio bovino. Esse tubo valvulado é preparado suturando-se o retalho de pericárdio externamente e, depois, invertendo-se o tubo, a válvula fica seu interior. Recentemente, reoperamos uma criança e, agora, aos 3 anos de idade, tanto o tubo, quanto a válvula estavam calcificados e foram substituidos por um outro, sem válvula. O Dr. Miguel Barbero-Marcial propôs uma técnica para os casos de truncus, evitando o tubo. Faz secção da parede anterior da artéria pulmonar e fecha a comunicação com a aorta com pequeno remendo. A secção longitudinal da via de saida chega ao limite superior. A artéria pulmonar é suturada diretamente na ventriculotomia, formando a parede posterior do "anel pulmonar". Uma placa de pericárdio com uma monocúspide completa a reconstrução da via de saida do ventrículo direito. Em alguns casos de aneurismas de aorta, usamos o pericárdio aberto suturado nos dois cotos e, quando se terminam as suturas proximal e distal, completa-se o tubo suturando, longitudinalmente, as duas bordas do pericárdio. Mais uma vez, cumprimento os autores pelo trabalho aqui apresentado.

\section{PROF. CARLOS MORAES (Encerrando)}

Eu queria agradecer aos comentadores, pelas palavras e fazer algumas considerações sobre os comentários. Todos nós conhecemos a velha polêmica, como o Dr. Ross reclama para si a denominação de operação de Ross; eu mesmo já o vi fazer este tipo de comentário e, por isto mesmo, tanto no trabalho escrito, como aqui, usei a seguinte terminologia: é usual; e é usual mesmo, e será sempre considerada a operação de Rastelli; jamais essa operaçāo será denominada operação de Ross, por mais que ele reclame; eu penso que a operação pioneira, que se denomina, no meu entender, operação de Rastelli, é toda operação que usa um conduto extracardiaco para se conectar o ventrículo venoso á circulaçāo pulmonar, e isto foi feito por Rastelli pela primeira vez, é indiscutivel. Mencionei, tanto no título deste trabalho, como na última conclusão, que esta era uma experiência inicial e não pretendemos tirar nenhuma conclusão definitiva; o objetivo deste trabalho, na realidade, é divulgar, entre os colegas, a técnica de preparação do tubo, referir que esse tubo, inclusive, já se encontra industrializado e à disposição de outros colegas e, no momento, o nosso objetivo é apenas este. Com relação aos elogiosos comentários do Dr. José Aldrovandro, eu agradeço; reconheço bem a experiênia do "Dante Pazzanese", ela está citada no nosso trabalho; apenas acho - e esta é uma experiência pessoal nossa - que, em algumas formas de cardiopatia onde existe hipertensão pulmonar e onde as paredes da artéria pulmonar são grossas, a sutura do tubo de Dacron não implica em maiores problemas; em alguns formas de atresia pulmonar, porém, com artérias pulmonares muito finas, a sutura do Dacron a essas artérias pulmonares implica, muitas vezes, em problemas; a artéria pulmonar rasga-se; então, eu penso que o pericárdio tem esta grande vantagem; ademais, a técnica usada e descrita por Joāo Bosco Oliveira tem, na minha opiniāo, a desvantagem de o 
MORAES, C. R.; RODRIGUESL, J. V.; GOMES, C. A.; MARINUCCI, L.; SANTOS, C. L.; COELHO, T. C.; CAVALCANTI, I. L. - Operaçāo de Rastelli utilizando-se conduto valvulado de pericárdio bovino: experiência inicial. Rev. Bras. Cir. Cardiovasc., 3(2): 84-92, 1988.

tubo ter que ser preparado na hora, ao passo que esses tubos de pericárdio já estão prontos, em diversos diâmetros e em diversos tamanhos. Eu agradeço, também, as palavras do Braile, bem como agradeço a grande contribuiçāo que ele deu à cirurgia cardiaca no Brasil, a partir do momento em que começou a estudar a conservação do pericárdio bovino; na realidade, tudo o que se faz de pericárdio bovino, no Brasil, tem origem em
São José do Rio Preto. A técnica do Miguel BarberoMarcial, mencionada, hoje, pelo Prof. Zerbini e citada pelo Prof. Adib, eu nāo conhecia, realmente; ela é engenhosa, mas, evidentemente, nāo pode ser aplicađa a todas as formas de cardiopatias nas quais a operação de interposição de um conduto está indicada, mas, dificilmente, neste tipo de truncus que nós mostramos, ela poderia ser aplicada. Muito obrigado. 